\title{
Correction to: A fuzzy rate controller for variable bit rate video using foveated just-noticeable distortion model
}

\author{
Mehdi Rezaei $^{1} \cdot$ Effat Karimghasemi $^{2}$
}

Published online: 14 October 2017

(C) Springer Science+Business Media, LLC 2017

\section{Correction to: Multimed Tools Appl (2017) 76:1439-1453 \\ https://doi.org/10.1007/s11042-015-3110-7}

In the original publication, the affiliations of the two authors were incorrectly written as "University of Sistan and Bluchestan" when it should be "University of Sistan and Baluchestan." The correct affiliations are presented in this erratum.

The online version of the original article can be found at https://doi.org/10.1007/s11042-015-3110-7.

Mehdi Rezaei

mehdi.rezaei@ece.usb.ac.ir

Effat Karimghasemi

ekarimghasemi@yahoo.com

1 Department of Electrical \& Computer Engineering, University of Sistan and Baluchestan, Zahedan, Iran

2 Signal Processing Lab, Faculty of Electrical and Computer Engineering, University of Sistan and Baluchestan, Zahedan, Iran 